\section{POTENCIANDO EL DESARROLLO LOCAL DE COMUNIDADES AFECTADAS POR DESASTRES ${ }^{1}$}

Alicia Cristina Razeto Pavez ${ }^{2}$

\section{Resumen}

En este artículo formularemos un análisis conceptual para proponer que los desastres usualmente conocidos como "naturales" son fenómenos sociales. Revisaremos cada fase de un desastre para descubrir las relaciones sociales que allí se generan y nos permiten fundamentar esta forma de comprenderlos. Como consecuencia de esta conceptualización, veremos que las políticas de respuesta a desastres no solo tienen que centrarse en la reconstrucción, sino que deben contemplar mecanismos para potenciar las condiciones de desarrollo de las zonas afectadas. En sintonía con esta perspectiva integral de abordaje de los desastres, se encuentra

\section{PROMOTING THE LOCAL DEVELOPMENT OF DISASTER-AFFECTED COMMUNITIES ${ }^{1}$}

Alicia Cristina Razeto Pavez ${ }^{2}$

\section{Abstract}

In this paper, we formulate a theoretical analysis to propose that the so-called "natural" disasters are in fact a social phenomena. Each phase of a disaster is analyzed so as to reveal the generation of social relationships and thus support this way to understanding them. As a consequence of this conceptualization, it is possible to see that policy responses to disasters do not only have to focus on reconstruction, but should include instruments that promote the development conditions of stricken areas. In line with this integral perspective to address 
el enfoque de desarrollo local. Mediante su revisión, hallaremos que son cinco las dimensiones a través de las cuales se puede aportar al desarrollo de las localidades afectadas por desastres.

PALABRAS CLAVE: DESASTRES, FENÓMENOS SOCIALES, RECONSTRUCCIÓN, DESARROLLO LOCAL.

Fecha de recepción: 29.06.12

Fecha de aceptación: 31.01.13

1 El artículo se enmarca en una investigación Fondecyt N¹110466, "Resiliencia y desarrollo local: de cara al desastre natural".

2 Chile. Trabajadora Social. Magíster en Gestión y Políticas Públicas de la Universidad de Chile. Académica de la Escuela de Trabajo Social, Facultad de Ciencias Sociales, Pontificia Universidad Católica de Chile.Email: arazeto@uc.cl

112 revista invi № 77 / Mayo 2013 / Volumen № 28: 111-136 disasters is the local development approach. The analysis of the latter provides five dimensions from which it is possible to contribute to the improvement of disaster-affected localities.

KEYWORDS: DISASTERS, SOCIAL PHENOMENA, RECONSTRUCTION, LOCAL DEVELOPMENT.

Received: 29.06 .12

Accepted: 31.01 .13

This paper is part of the Fondecyt research N¹110466 "Resilience and Local Development: Facing the Natural Disaster."

2 Chile. Social Worker. MA in Management and Public Policy, University of Chile. Academic, School of Social Work, Faculty of Social Sciences, Pontifical Catholic University of Chile. Email: arazeto@uc.cl

ARTICLE: Promoting the local development of disaster-affected communities /

Alicia Cristina Razeto Pavez 


\section{Introducción}

A dos años del terremoto y tsunami del 27 de febrero, hoy parece haber consenso en que el país no estaba completamente preparado para afrontar un desastre de gran magnitud. Esto se demostró rápidamente en la falla de los sistemas de alerta de maremoto, del sistema de comunicaciones y del control del orden público durante la emergencia ${ }^{3}$. Posteriormente, se ha demostrado en las dificultades del proceso de reconstrucción, ya que a pesar del tiempo transcurrido, las localidades más afectadas aún intentan levantarse y la reconstrucción todavía no muestra resultados en todas las zonas afectadas ${ }^{4}$.

A los problemas generados por el desastre, se suma como antecedente un escaso desarrollo teórico en relación a las estrategias más adecuadas para comprenderlo y abordarlo de manera efectiva e integral. Si bien las ciencias físicas y naturales han contribuido al estudio de los fenómenos naturales, no ha sucedido lo mismo con las ciencias sociales, que han aportado de manera insuficiente al estudio de la materia en Chile y el resto de América Latina ${ }^{5}$. Esto es contradictorio desde dos puntos de vista. El primero es que somos uno de los países más sísmicos del planeta, por lo tanto, altamente

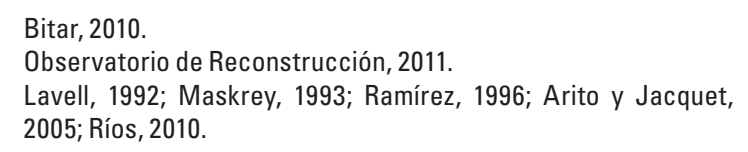

vulnerables a terremotos y tsunamis. El segundo es que los desastres causan un gran y complejo impacto en las sociedades, produciendo un cataclismo social e institucional ${ }^{6}$. De allí que sea necesario desarrollar estudios sociales para interpretarlos, prevenirlos y resistirlos.

Este artículo tiene como finalidad contribuir a la reflexión conceptual sobre el tema de los desastres. Especialmente, aportar con una perspectiva integral para la comprensión de los desastres y para la formulación de políticas y programas públicos de intervención en localidades afectadas en escenarios post-desastre. Dicha finalidad está en sintonía con el Banco Interamericano de Desarrollo ${ }^{7}$ y otros autores $^{8}$ que nos advierten sobre la necesidad de contar con enfoques más integrales para anticiparse a los desastres, que permitan la reducción de los riesgos y la recuperación posterior.

En el ámbito de la comprensión, proponemos que los desastres, como terremotos y tsunamis, no son solo eventos de carácter natural sino también fenómenos sociales ${ }^{9}$. En el ámbito de las estrategias de acción, postulamos que los programas públicos no solo deben centrarse en la emergencia y reconstrucción material de las comunidades afectadas,

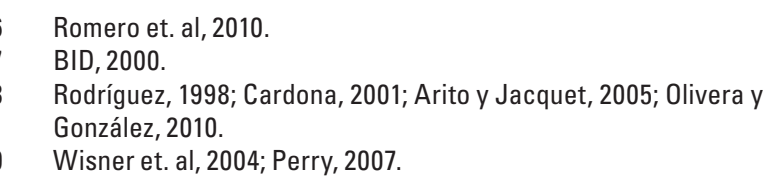


sino también en generar las condiciones para que esas localidades puedan encaminarse en procesos de desarrollo local. En esta parte describiremos el enfoque de desarrollo local y sus múltiples dimensiones, de modo de contribuir a visualizar las diversas aristas que las políticas y programas de respuesta a desastres podrían atender.

\section{Desastres como fenómenos sociales}

Los terremotos y tsunamis usualmente son catalogados como desastres naturales. La connotación de natural obedece al origen de estos eventos, como es en el caso de los terremotos, el movimiento de las placas de la corteza terrestre. Esta forma de denominarlos, si bien es congruente en relación a la procedencia de los desastres, es limitada en el sentido que excluye los efectos y consecuencias sociales. Ante esto, surgen las siguientes interrogantes: ¿Por qué denominamos de esta manera a los desastres? ¿Cuál es la diferencia entre la mirada natural y social sobre estos fenómenos?

Tras esta forma de calificar a los desastres está el gran protagonismo que asume el suceso en el momento de su ocurrencia, por su magnitud y características. El protagonismo que ocupa el desastre se explica por lo inesperado, impredecible, intenso

114 revista invi № 77 / Mayo 2013 / Volumen № 28: 111-136 y traumático que puede llegar a ser. Los desastres nos recuerdan lo indomable que es la naturaleza, nos invitan a reconocer que nuestro conocimiento sobre el planeta es incompleto e incierto ${ }^{10}$ rompiendo así, al menos temporalmente, el predominio de la lógica de control y poderío que el ser humano históricamente ha impuesto sobre ella. Desastres como los terremotos y tsunamis, se producen por la acción de la naturaleza, por lo tanto es consustancial que el paradigma predominante en su estudio sean las ciencias naturales y físicas. Los estudios provenientes de dichas ciencias intentarán comprender las causas y las formas en las que se manifiesta; explicar y predecir su ocurrencia, de modo de prevenir o controlar los efectos causados por este tipo de sucesos. A través del uso del método científico, las investigaciones de estas disciplinas han logrado un reconocimiento por la academia y comunidades de expertos, desarrollando conocimiento legitimado y validado culturalmente.

Ante la segunda pregunta planteada, podemos señalar que la diferencia que hay entre comprender a los desastres como exclusivamente naturales o como fenómenos sociales es abismante. Concebir a los terremotos y tsunamis solo como sucesos naturales, excluye la enorme complejidad de la relación que hay entre sociedad y naturaleza, restringiendo la comprensión del fenómeno y potenciales acciones de respuesta. Nosotros proponemos que

10 Mitchell, 1999 
los desastres si bien tienen un origen natural, son fenómenos profundamente sociales ${ }^{11}$. Tal como lo señala Kreps ${ }^{12}$, los desastres pueden ser catalogados como fenómenos sociales porque son un acontecimiento histórico dramático y porque obligan a la generación de acción colectiva, es decir, los desastres son catalizadores sociales. En este sentido, recogiendo los planteamientos de Cardona ${ }^{13}$ sobre la concepción de riesgo desde el punto de vista de los desastres, creemos necesario adoptar perspectivas integrales para comprender la forma en la que estos impactan y transforman a la sociedad.

En las conceptualizaciones sobre desastres, encontraremos que la mayoría de ellas los define como tal por el impacto producido en las sociedades, en los individuos, territorios, economía y medioambiente. A continuación presentamos ciertas definiciones de desastres y distinciones que precisan el concepto:

- La palabra desastre proviene de la raíz latín astronómico o astrológico dis y astro, cuyo significado es "separado de las estrellas"14. El origen de esta palabra, sugiere entenderlo como un evento que ocurre por una configuración astrológica desafortunada. Más contemporáneamente, nos referimos a un desastre cuando un riesgo es presentado o materializado en una

\footnotetext{
11 Dynes, 1988; Perry, 2007

12 Kreps, 1998.

13 Cardona, 2001

14 Coppola, 2011.
}

comunidad. La idea del riesgo no es igual a la del desastre, ya que el primero se refiere a algo que aún no acontece, en cambio desastre se refiere a un evento que ya ocurrió ${ }^{15}$. Tampoco es lo mismo hablar de evento y desastre, ya que este último es el que logra sobrepasar la capacidad de la comunidad para abordarlo.

- Desde la antropología, se define desastre como un "proceso que involucra una combinación de agentes potencialmente destructivos -los que pueden ser naturales o provenir del ambiente tecnológico- y una población en una condición de vulnerabilidad producida social y tecnológicamente"16. La combinación de ambos componentes produce daño o pérdida de los principales elementos de la organización social y de las instalaciones físicas de una comunidad al grado que las funciones esenciales de la sociedad son interrumpidas o destruidas, lo que genera stress individual y desorganización social de distinta gravedad ${ }^{17}$.

- Según la Estrategia Internacional para la Reducción del Desastre de las Naciones Unidas ${ }^{18}$, un desastre es una seria interrupción en el funcionamiento de una comunidad o sociedad que ocasiona una gran cantidad de muertes al igual

15 Stallings, 1997.

16 Oliver-Smith, 1996, p. 303.

17 Ibíd.

18 EIRDNU, 2009 
que pérdidas e impactos materiales, económicos y ambientales que exceden la capacidad de la comunidad o la sociedad afectada para hacer frente a la situación mediante el uso de sus propios recursos.

- De acuerdo a Lavell ${ }^{19}$, un desastre "es una ocasión de crisis o estrés social, observable en el tiempo y el espacio en que sociedades o sus componentes (regiones, comunidades) sufren daños o pérdidas físicas y alteraciones en funcionamiento rutinario. Tanto las causas como las consecuencias de los desastres son productos de procesos sociales que existen al interior de una sociedad".

Considerando este conjunto de definiciones, vemos que la característica más marcada de los desastres es que producen daños en las personas y en el funcionamiento de la sociedad. Este rasgo les imprime la connotación más evidente en su categorización como fenómenos sociales. Sin embargo, también es preciso reparar en que la sociedad y la acción de los individuos pueden incidir en la ocurrencia de los desastres. De acuerdo a McCaughey ${ }^{20}$. las decisiones tomadas por el ser humano influyen en los resultados de los desastres, sean estos de origen natural o antropogénico. En síntesis, comprender a los desastres como fenómenos sociales genera una diferencia significativa con la perspectiva puramente naturalista, tanto a nivel de las estrategias

19 Lavell, 1993, p. 80.

20 McCaughey et. al, 1994.

116 revista invi № 77 / Mayo 2013 / Volumen № 28: 111-136 de prevención como del manejo de las situaciones post-desastre. Se trata de una perspectiva más integral que produce resultados más efectivos para las comunidades que los han sufrido. Al ser considerado como fenómeno social, la perspectiva integral advierte sobre la presencia de una situación anterior que sobredetermina al desastre y una situación posterior al evento que forma parte ineludible en su categorización. En esta línea, visualizamos que el desastre no es solo un estado o situación sino que es un proceso compuesto de momentos o fase ${ }^{21}$, dinámicas y relaciones sociales, como veremos en cada uno de los siguientes elementos.

\section{Fase previa al desastre}

El momento previo influye fuertemente en las características y consecuencias de un desastre. Ahora nos detendremos en las características de las relaciones entre individuos, sociedad y naturaleza de esta fase, que hacen del desastre un fenómeno social.

\section{EFECTOS DE LA ACCIÓN DEL SER HUMANO SOBRE LA NATURALEZA}

Los desastres, antropogénicos o tecnológicos ${ }^{22}$, son causados por los efectos directos de la acción de los individuos sobre la naturaleza. Es el caso de

\section{Vargas, 2002}

22 Lavell, 1996. 
los derrames de compuestos químicos en el mar, incendios, explosiones químicas, nucleares, etc. Otros desastres de origen natural, cada vez más han sido asociados a los efectos de la acción humana, vinculándolos al crecimiento vertiginoso de las industrias y contaminación, que producen gases invernadero y aerosoles que afectan la temperatura del planeta provocando el cambio climático ${ }^{23}$. Este cambio impacta profundamente los equilibrios del macrosistema propiciando la ocurrencia de desastres, como son las inundaciones por efecto de las lluvias, aluviones, sequías, ondas de calor, huracanes, entre otros ${ }^{24}$. En cierto sentido, el cambio climático pone en peligro el logro de los objetivos del desarrollo, por los impactos que produce sobre el medioambiente $e^{25}$.

\section{AMENAZAS Y PELIGROS NATURALES IGNORADOS POR EL SER HUMANO}

El rápido y explosivo crecimiento de las ciudades no siempre ha respetado los riesgos presentes en el medio natural. Según Lavell ${ }^{26}$, los riesgos suponen la existencia de dos factores vinculados entre sí: amenazas, entendidas como la probabilidad de ocurrencia de un evento físico dañino, y vulnerabilidad, concebida como propensión de una sociedad

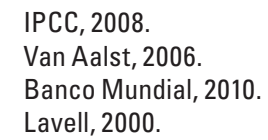

a sufrir daños, en ciertos contextos urbanos. El veloz crecimiento de las ciudades las ha dejado más expuestas a peligros naturales y desastres ${ }^{27}$, volviendo a éstos más mortales y frecuentes ${ }^{28}$. Aún más, cuando la urbanización no responde a una planificación que considere las amenazas y vulnerabilidades ni tampoco desarrolla una adecuada fiscalización del cumplimiento de la normativa de prevención y mitigación de riesgos.

Las situaciones de riesgo son preexistentes a los desastres y condicionan enormemente la magnitud de los daños que ocasionan. Determinar en qué medida se toman en cuenta y se regulan estos elementos, depende de las decisiones expresadas a través de la legislación y de las políticas públicas, aspecto de carácter político y sociocultural.

\section{POLÍTICAS DE PREVENCIÓN Y MANEJO DE DESASTRES}

El continente americano comparte junto a Asia los primeros lugares en la ocurrencia de desastres, sumando en su conjunto más del 60\% del total de desastres registrados en el mundo ${ }^{29}$. Ante datos como este, pareciera evidente que formular políticas y estrategias para la prevención y manejo de los desastres sea hoy un elemento fundamental de las

27 Mitchell, 1999; Quarantelli, 2001.
28 Tucker et. al, 1994.

29 García, 2008. 
políticas públicas de los países latinoamericanos ${ }^{30}$ Sin embargo, podemos constatar que al menos en Chile este tema aún no se transforma en un asunto prioritario para la agenda pública ${ }^{31}$.

Las políticas de prevención, preparación y mitigación del riesgo forman parte del momento previo al desastre, ya que en el mejor de los casos, han sido formuladas anteriormente como resultado del aprendizaje de un desastre. Las políticas manifiestan la forma en la que se comprende la relación entre sociedad y naturaleza, ya que contiene los modos de interpretar y gestionar a los desastres. La existencia de políticas de prevención, mitigación y respuesta pueden ayudar enormemente a disminuir los daños ocasionados por un desastre, sobre todo si contemplan la participación activa de los habitantes de los territorios azotados. Más aun, pueden hacer la diferencia entre si lo que se desencadena termina siendo solo un evento o un desastre. En este sentido la consideramos como otra de las características de este momento, que al ser resultado de decisiones humanas, expresan una cierta relación entre sociedad y naturaleza, haciendo de los desastres un fenómeno social.

30 Vargas, 2002; Cepal, 2000

31 Naciones Unidas, 2010

118 revista invi № 77 / Mayo 2013 / Volumen № 28: 111-136

\section{Fase durante y posterior al desastre}

Este momento constituye la cúspide del desastre, puesto que concita la mayor atención, interés y preocupación de la sociedad, principalmente de sus afectados y testigos. Tal como señalamos, un desastre se califica como tal por sus consecuencias en relación a las pérdidas humanas, económicas y ambientales. Todas estas ocurren en el momento en que éste se genera y se cuantifican después que ha sucedido. Los elementos de esta fase que ayudan a comprender la naturaleza social del fenómeno son:

\section{REACCIONES DE LOS INDIVIDUOS ANTE UN DESASTRE}

La conducta es la reacción visible que tienen las personas ante un desastre. Esta se encuentra fuertemente mediada por la educación que reciban en el ámbito de cómo afrontar las emergencias. La educación modela las reacciones y comportamientos, transformándolos en aliados para la minimización 
de los daños. Además, la cultura de las poblaciones en riesgo sirve para reaccionar y resistir frente a las amenazas $^{32}$. Por ejemplo, ante la ocurrencia de un tsunami originado por un sismo de grandes proporciones, marca una enorme diferencia el hecho que la población escape hacia las lomas o hacia la zona costera. O por ejemplo, conocer el riesgo sísmico del material de construcción de una vivienda es fundamental para saber cómo actuar frente a un terremoto.

El comportamiento también es mediado por la intuición y emociones suscitadas ante la catástrofe. Por cierto, las acciones individuales pueden verse altamente influidas por las acciones colectivas en momentos de pánico y descontrol. Por eso podemos señalar que las reacciones son el resultado de un contexto social y de relaciones sociales, que pueden llegar a marcar un impacto en la magnitud de los daños.

\section{CAOS Y DESCONTROL SOCIAL PRODUCTO DE LA EMERGENCIA}

Las situaciones de destrucción y emergencia causan pánico y pueden llegar a configurar un escenario temporal de caos, descontrol y desconfianza social, propicias para la producción de saqueos y robos $^{33}$. Así, los desastres pueden producir una alteración transitoria y notable en el desarrollo de las relaciones sociales que impactan el funcionamiento de la sociedad. La inseguridad e incertidumbre que se generan en una situación de descontrol ocasionada por un evento que pone en jaque el orden natural, pueden desestabilizar las relaciones sociales en las cuales la desconfianza hace que los individuos sean vistos como amenaza a la seguridad personal. Este tipo de relaciones sociales le imprimen una mayor connotación de gravedad a los desastres, agudizando muchas veces los daños materiales e inmateriales y volviendo necesaria la intervención militar de los territorios afectados para retomar el orden.

\section{SOLIDARIDAD Y COOPERACIÓN COMO RESPUESTA A LA EMERGENCIA}

Las relaciones sociales no solo pueden ser caóticas, sino también tremendamente positivas entre quienes han sido afectados por el desastre y también por quienes han sido testigos. En las muestras de cooperación y solidaridad se hacen patentes los mejores valores culturales de una sociedad que ha sido azotada por una calamidad. Estas iniciativas pueden ser transversales a distintos sectores de la sociedad, o surgir especialmente desde la sociedad civil, como es el caso del voluntariado

32 Aguirre, 2004.

33 Sanzana, 2010. 
de organizaciones sociales o grupos de jóvenes espontáneamente conformados para ayudar a las localidades.

Este tipo de iniciativas son favorecidas gracias a las tecnologías de información y comunicación, ya que a través de ellas los desastres generan profundo impacto a nivel nacional e incluso internacional. La cooperación y la solidaridad puede manifestarse más allá de las fronteras nacionales, como fue el caso de la ayuda humanitaria a dos días del terremoto en Chile ${ }^{34}$. De esta manera, los desastres originan relaciones de cooperación entre las personas, grupos y organizaciones sociales, y países enteros, abarcando sistemas sociales completos.

\section{DAÑOS Y COSTOS GENERADOS POR EL DESASTRE}

Un evento que no cause daños y pérdidas no será calificado como desastre. Como señala Oliver$S_{\text {Smith }}{ }^{35}$, estos afectan a una población vulnerable y alteran las funciones esenciales de la sociedad, excediendo su capacidad para hacer frente a la situación ${ }^{36}$. En este sentido, los desastres concitan la atención e interés público por sus consecuencias y los costos que implica en términos de vidas humanas, perjuicios al bienestar de la población y daño

34 López y Santana, 2011.

35 Oliver-Smith, 1996.

36 EIRDNU, 2009.

120 revista invi № 77 / Mayo 2013 / Volumen № 28: 111-136 económico. Los desastres azotan las economías de los territorios ${ }^{37}$ afectados en el corto y largo plazo ${ }^{38}$ y es uno de los más graves perjuicios, en conjunto con la pérdida de vidas humanas que pueden generar. De allí que un desastre puede poner en jaque no solo la relación entre economía y naturaleza, sino también entre economía y sociedad.

\section{EFECTOS DEL DESASTRE SOBRE LAS ORGANIZACIONES PÚBLICAS RESPONSABLES DE INTERVENIR}

Los impactos de los desastres abarcan a las personas y a las organizaciones que existen en las zonas afectadas ${ }^{39}$. Las capacidades institucionales de los organismos gubernamentales que ejecutan las políticas de recuperación son fundamentales para responder a los daños ocasionados. Estas tienen la responsabilidad no solo de reconstruir viviendas e infraestructura dañada, sino también de recuperar a la comunidad. A pesar que las organizaciones son afectadas por el desastre, deben ser actores protagónicos en la ayuda durante y después de la emergencia. Durante un desastre, las organizaciones enfrentan múltiples contingencias y deben cambiar sus rutinas ${ }^{40}$. Recibirán las demandas de los habitantes por la solución a sus problemas y serán

\footnotetext{
37 Quarantelli, 2002.

38 Cepal, 2000.

39 McCaughey et. al, 1994.

40 Quarantelli, 2002.
} 
objeto de cuestionamientos y altas exigencias, sobre todo cuando no ha existido una política clara de acción frente a este tipo de eventos. Las demandas que reciben por parte de los habitantes y de los organismos centrales las estimulan a generar nuevos diagnósticos, flexibilizar estrategias, establecer alianzas y, por sobre todo, generar aprendizajes y lecciones que contribuyan a perfeccionar las prácticas del futuro en nuevos desastres. Por ello, una situación de desastre puede alterar las formas de las relaciones establecidas entre individuos y organizaciones, propiciando relaciones de confianza o desconfianza, de cooperación o antagonismo, de paternalismo o empoderamiento social.

\section{Desde la reconstrucción hacia el desarrollo local}

Entender los desastres como fenómenos sociales acarrea consecuencias en la formulación de las políticas de recuperación. La principal premisa con la cual desarrollamos esta parte del artículo es que las respuestas frente a los desastres deben superar las visiones centradas solamente en la reconstrucción física y el asistencialismo ${ }^{41}$. Si bien este es un componente necesario de las políticas, es insuficiente para lograr que las localidades afectadas se sobrepongan a la situación de emergencia

41 Caputo y Herzer, 1987. y se encaminen hacia el desarrollo. Para pensar y formular las políticas de respuesta de desastres es necesario adoptar perspectivas integrales ${ }^{42}$ alineadas con la conceptualización del desastre como fenómeno social. Postularemos que el enfoque de desarrollo local es una perspectiva que sintoniza con esta visión porque pone en el centro a las personas y su bienestar, ofreciendo un gran potencial para superar las adversidades enfrentadas por las localidades.

El nexo conceptual entre desastre y desarrollo está en la noción de cambio. En este sentido, es preciso comenzar reconociendo la relación que hay entre desastre y cambio social ${ }^{43}$, consustancial a la noción de desastre como fenómeno social. Dicha relación se expresa en que una vez que un desastre afecta a una localidad, ésta nunca más volverá a su estado original, ya que se gatillan cambios en amplios y profundos sentidos, desde el plano personal-familiar de la comunidad que lo vive hasta el plano territorial como son los cambios en la configuración y ordenamiento urbano-espacial. Este es un punto muy relevante de considerar, puesto que tiene profundas implicancias para las políticas que se implementarán posteriormente. Por un lado, se hace necesario volver a estudiar el contexto social de las comunidades posterior al suceso, y por otro, es relevante asumir que ninguna política podrá

42 Herzer et. al., 2002; Rodríguez, 1998.

43 Audefroy, 2007; Oliver-Smith, 1995. 
hacer retornar a la localidad al mismo estado en el que estaba antes del desastre. Podemos enfatizar en que un desastre es un acontecimiento violento que irrumpe en las distintas formas de la vida social, transformando la vida cotidiana y las relaciones sociales ${ }^{44}$. Lo anterior produce un escenario nuevo que genera sentidas pérdidas, pero que también abre oportunidades, ya que por un lado, los daños hacen visible ante el país y mundo entero las necesidades que aquejan a las localidades, mientras por otro lado, un desastre revela las fuerzas de las localidades, siendo la capacidad de resiliencia una de las más importantes ${ }^{45}$.

En un contexto de desastre, las sociedades se encuentran más susceptibles al cambio, ya que el desorden es uno de sus mejores posibilitadores ${ }^{46}$. En este artículo, proponemos que este contexto de cambio es oportuno para recuperar las condiciones para el desarrollo local de los territorios. En este sentido, es preciso tener en cuenta que los desastres afectan la posibilidad de desarrollo futuro de las localidades, por lo que colocar el tema del desarrollo en el centro de los programas permite instalar una mirada sustentable de largo plazo ${ }^{47}$, que trascienda al foco de la emergencia. Por lo demás, implementar políticas de desarrollo permitirá generar las condiciones para que la localidad

\footnotetext{
44 Audefroy, 2007.

45 Cardona, 2001; Aguirre, 2004

46 Audefroy, 2007.
}

47 Smith y Wenger, 2007. revierta su vulnerabilidad a sufrir nuevos y futuros eventos, refundando un nuevo contexto territorial en el cual las comunidades adquieren mayor resiliencia para enfrentar futuros desastres.

Tal como lo señalara Arocena ${ }^{48}$ al dar cuenta del origen del concepto de desarrollo asociado a los países europeos después de la segunda guerra mundial: "cuando la construcción o la reconstrucción conoció sus límites, se comenzó a hablar de desarrollo". Nosotros proponemos que la reconstrucción, aunque es una estrategia urgente y necesaria para paliar los efectos materiales más visibles y directos de los desastres, tiene límites. Ante éstos, el enfoque de desarrollo abre oportunidades, ya que es más pertinente para retratar los principios y objetivos que los países modernos y democráticos buscan para sus habitantes. Así, las políticas de desarrollo de mediano y largo plazo debieran ser capaces de encauzar los procesos de cambio que están experimentando a raíz de la emergencia, conduciéndolas hacia un escenario que tenga como horizonte las mejoras de los niveles de bienestar de los habitantes de la localidad. De este modo, las localidades podrán adaptarse al nuevo escenario post desastre, y al mismo tiempo, acercarse a mejores parámetros de desarrollo.

48 Arocena, 2002, pág. 6 
No es objeto aquí sumergirnos en el amplio debate existente en la literatura sobre desastres y desarro$110^{49}$, ya que es otro el tema que nos importa: el descubrir las potencialidades que tiene el enfoque de desarrollo local para intervenir en localidades que fueron afectadas por desastres. Dentro de los diversos enfoques de desarrollo, destacamos la potencialidad que tiene el enfoque de desarrollo local para comunidades afectadas por desastres, puesto que está circunscrito a una escala territorialmente bien delimitada, se trata de un desarrollo localmente situado.

Tomando como referencia la definición de Gallicchio y Camejo ${ }^{50}$, por desarrollo local entenderemos:

- Un proceso impulsado por una concertación de agentes, públicos y privados, que interactúan en un territorio.

- Donde participan activamente los habitantes de la localidad en la creación de un proyecto común de desarrollo.

- Dicho proyecto común de desarrollo implica no solo crecimiento económico, sino que también integración socio cultural y equidad.

- Es un proceso que resguarda la sostenibilidad del medioambiente, y cuyos principios son

49 Stephenson, 1991; OEA, 1991; Mansilla, 1993; Lavell, 2000; Cepal, 2010.

50 Gallicchio y Camejo, 2005 afines con la igualdad entre los géneros y el equilibrio territorial.

- Su finalidad es contribuir al bienestar de los habitantes de un territorio, de modo de incorporarlos al desarrollo del país y a los cambios de la economía internacional.

El enfoque de desarrollo local tiene ciertos principios básicos ${ }^{51}$, entre los que están la centralidad de las personas en los procesos de desarrollo, a quienes se les concibe como el motor y al mismo tiempo el objetivo del desarrollo El valor de las personas y su realización personal es una piedra angular sobre la cual se construyen las estrategias de desarrollo. La centralidad de las personas sintoniza con los contextos de desastres, ya que éstas son las principales afectadas y al mismo tiempo el principal motor del que dispone el territorio para superar la adversidad. Otro de los principios del enfoque es el reconocimiento de la riqueza y el enorme potencial que tienen las iniciativas locales de desarrollo. Más que depender de instituciones centrales o de los sistemas macroeconómicos, que merman la capacidad colectiva endógena, se intenta promover los proyectos que surgen desde la localidad, con el objetivo de generar empleo, capital social y humano ${ }^{52}$. En un escenario de desastre,

51 Vachon, 2001

52 Ibíd 
este principio cobra gran relevancia si se considera que la mayor cantidad de empleo es generado por los microemprendimientos o por grandes empresas locales. Al mismo tiempo, los recursos naturales y humanos de las localidades son insumos fundamentales para impulsar estas iniciativas.

La potencialidad del enfoque de desarrollo local depende del reconocimiento de la heterogeneidad que hay al interior y entre los distintos territorios azotados por desastres. De este modo, es fundamental contar con información que permita tomar las decisiones correctas para encauzar a cada localidad en el camino de avanzar hacia su propio potencial de desarrollo. En otras palabras, no hay solo un esquema ni un modo de avanzar hacia el desarrollo. El enfoque de desarrollo local asumirá que cada territorio, de acuerdo a sus propias especificidades, necesidades y capacidades, tiene distintos umbrales y potenciales de desarrollo local.

Así, vemos que el desarrollo local como enfoque para la formulación de políticas de respuesta ante desastres, es pertinente y oportuno desde el momento en que concebimos los desastres como fenómenos sociales. A diferencia de las políticas puramente centradas en la reconstrucción, el desarrollo local considera a la reconstrucción como uno de los momentos, nunca el único, del proceso de

124 revista invi № 77 / Mayo 2013 / Volumen N ${ }^{0} 28$ : 111-136 recuperación de la comunidad ante los efectos adversos del desastre. En el siguiente apartado identificaremos las principales dimensiones de este enfoque, aplicándolas a los contextos de desastre e implementación de políticas de respuesta.

\section{Enfoque multidimensional de desarrollo local para políticas de respuestas a desastres}

El concepto de desarrollo local puede resultar un tanto ambiguo ${ }^{53}$ al momento de intentar aplicarlo como enfoque para las políticas post-desastre. En un contexto de crisis, el desarrollo local puede parecer un horizonte lejano en lugar de un objetivo y estrategia de acción de los programas implementados. En vista de ello, es preciso que realicemos una operacionalización del enfoque, en términos de identificar las dimensiones que lo componen y que puedan orientar la formulación de las políticas de respuesta ante desastres. Gallicchio y Camejo ${ }^{54}$ señalan que hay cuatro dimensiones que componen el concepto de desarrollo local de un territorio. Estas son la dimensión económica, sociocultural, política y medioambiental. En este artículo utilizaremos la propuesta de los autores, pero dividiremos la sociocultural en dos dimensiones distintas.

53 Furió, 1994.

54 Gallicchio y Camejo, 2005.

ARTíCULO: Potenciando el desarrollo local de comunidades afectadas por desastres /

Alicia Cristina Razeto Pavez 
Creemos que la dimensión social y cultural si bien están estrechamente vinculadas, cada una es tan compleja e importante para el desarrollo local, que conviene separarlas, sobre todo en contextos complejos como los originados tras un desastre.

De aquí en adelante describiremos las dimensiones y las analizaremos en función del desarrollo de políticas post-desastre en territorios afectados. Las cinco dimensiones del desarrollo local son las siguientes:

\section{ECONÓMICA}

A pesar de ser uno de los motores relevantes para el desarrollo de una localidad, la economía es uno de los sectores más afectados por un desastre. Desde el enfoque del desarrollo local, el eje central de esta dimensión es la creación, acumulación y distribución de la riqueza dentro de un territorio ${ }^{55}$. Es importante enfatizar la importancia de estos tres elementos, por cuanto la distribución de los recursos se vuelve tan importante como su creación y acumulación. En este enfoque, la distribución no forma parte de las responsabilidades de las políticas sociales, sino que es parte fundamental del funcionamiento de la economía de una localidad. Si bien la creación y acumulación de riquezas es clave para elevar el bienestar de parte de la población, éstas deben estar distribuidas entre sus

55 Ibíd. habitantes y no solo entre unos pocos. Esto marca una diferencia de las visiones más tradicionales de desarrollo como crecimiento económico, donde solo ciertos grupos sociales se enriquecen.

Uno de los elementos más relevantes dentro de esta dimensión corresponde a la existencia y fortalecimiento de las iniciativas económicas locales (IEL). Dentro de las IEL se encuentran los microemprendimientos, las pequeñas, medianas y grandes empresas que operan al interior del territorio. Las IEL son las generadoras de empleo para los habitantes de la localidad, transformándose en las productoras más directas de riquezas para el conjunto de la población. Desde la visión del desarrollo económico local, las IEL consideran en su producción y gestión los valores territoriales de identidad, diversidad y flexibilidad. De este modo, las IEL funcionan vinculadas a su entorno territorial ${ }^{56}$.

La economía de una localidad temporalmente se inmoviliza puesto que la destrucción de infraestructura y equipamiento del sector industrial, comercio y servicios imposibilitan su funcionamiento. En consecuencia, el empleo también resulta perjudicado. En esta dimensión, las políticas de respuesta ante un desastre observarán los grados de destrucción de la infraestructura y equipamientos de las principales IEL, tanto de las que se desarrollan a una microescala como las que lo hacen a una escala

56 Alburquerque, 2004. 
mayor. Al mismo tiempo, desplegarán programas, subsidios y créditos, para lograr el restablecimiento pronto del funcionamiento de las IEL, de modo tal que el empleo de la localidad no se vea tan fuertemente afectado. Por otra parte, las políticas de respuesta ante los desastres, formularán programas para la creación y fortalecimiento de nuevas IEL como también de apoyo a las ya existentes, para fomentar los mercados de comercialización y especialmente, para vislumbrar los modos mediantes los cuales las IEL pueden generar estrategias solidarias para el apoyo de las localidades afectadas. Por su parte, el municipio, que es el principal agente de desarrollo local, impulsará la creación de un marco regulatorio pertinente a la situación de desastre, orientado a facilitar el funcionamiento de las IEL, promoviendo y premiando aquellas iniciativas solidarias. Hay que considerar que si bien un desastre tiene consecuencias económicas dañinas para la región que lo experimenta, también puede abrir oportunidades en términos de aumentar la demanda interna de bienes y servicios que tienen relación con la reposición de lo destruido y la progresiva reconstrucción (CEPAL, 2010). Es en dicha oportunidad donde las políticas de respuesta ante desastres pueden contribuir a que las IEL asuman un mayor papel en la dinamización de la economía local.

126 revista invi № 77 / Mayo 2013 / Volumen № 28: 111-136

\section{SOCIAL}

Las relaciones sociales al interior de un territorio son articuladoras e impulsoras de los procesos de desarrollo de una localidad. Al igual que la economía, el desarrollo social de una comunidad es fundamental para resistir y sobreponerse a una situación de desastre. Al mismo tiempo, éste puede transformarse en un factor de desarrollo social ${ }^{57}$ si se estimula la participación de la comunidad en la resolución de los problemas suscitados. Desde el enfoque del desarrollo local, las personas son el centro del desarrollo, por lo cual debiesen ser los principales actores de las políticas de respuestas a desastres afines a este enfoque. La centralidad de las personas en los procesos de desarrollo, llevan a primera plana las mejoras de la calidad de vida de los habitantes de un territorio, así como también los avances en términos de integración social ${ }^{58}$. En consecuencia, la pobreza, la desigualdad y la exclusión social son barreras que entorpecen el desarrollo local.

Bajo este enfoque, la acción colectiva es deseable por sobre la acción individual. Varios autores ${ }^{59}$ han coincidido en destacar la importancia del capital social para construir el desarrollo de los territorios. La asociatividad, redes y relaciones de confianza entre los habitantes, organizaciones comunitarias

57 Pliego, 1992

58 Gallicchio y Camejo, 2005.

59 Trigilia, 2001; Gallicchio, 2004; Allen, 2006; Alcañiz, 2008; Madoery, 2001; Andre y Rego, 2003.

ARTíCULO: Potenciando el desarrollo local de comunidades afectadas por desastres /

Alicia Cristina Razeto Pavez 
y otros organismos públicos y privados son un factor fundamental para la creación de un proyecto colectivo. En conjunto, pueden ser los protagonistas de la implementación y del control de las estrategias acordadas. De este modo, la participación y organización de la comunidad es indicativa del desarrollo social de la localidad, en términos de su capacidad para hacerse responsable y protagonista de su propio proceso de mejora. Las políticas postdesastre en todas sus expresiones, deben ser capaces de incorporar y estimular la participación y organización de la comunidad para la formulación e implementación de las políticas. Recordemos que después de un desastre, las comunidades generan múltiples iniciativas colectivas de solidaridad y cooperación, por lo cual es fundamental que los municipios sean capaces de facilitar su funcionamiento para hacerlas sostenibles en el tiempo. Las iniciativas solidarias y cooperativas aportan positivamente no solo a paliar los efectos dañinos de un desastre sino también a producir capital social. De mantenerse en el tiempo, este tiene un enorme efecto en el potencial desarrollo de las localidades. Lamentablemente, las experiencias tienden a mostrar que solo pocas iniciativas perduran.

Asimismo, las políticas post-desastre colocarán especial énfasis en la observación y reversión de los daños a la infraestructura social, como las sedes vecinales, entre otros, de modo de contribuir al encuentro colectivo entre los habitantes y la generación de nuevas iniciativas. Por otra parte, las políticas post-desastre crearán recursos especiales para apoyar y fortalecer a las organizaciones sociales, incorporándolas a los procesos de toma de decisiones y formulación de programas sociales para cubrir las necesidades de la población, las que habrán cambiado en comparación con la situación previa al desastre. Estas no solo consistirán en necesidades de vivienda, sino también en las relativas a salud, educación, seguridad, empleo, entre otras.

\section{CULTURAL}

La cultura influye en cómo las sociedades interpretan y actúan frente a un desastre. Entendemos por cultura una construcción social portadora de la identidad del territorio, en base a su historia, tradiciones, normas y valores sociales. La cultura abarca e influye en todas las actividades de los habitantes del territorio ${ }^{60}$, constituyéndose como el eje de todo proceso de desarrollo. Los valores tienen sus raíces en la cultura, como lo son la solidaridad, el altruismo, el respeto y la tolerancia, los que son esenciales para lograr un desarrollo local sustentable. Así, los valores de los cuales es portadora una sociedad local van a incidir fuertemente en las formas y metas del desarrollo ${ }^{61}$.

60 Figueroa Diaz, 2002

61 Ibíd. 
Al favorecer la cohesión social, la cultura es fundamental para la construcción del tejido social ${ }^{62}$, y como hemos visto, este es indispensable para la formulación de un proyecto colectivo y compartido de desarrollo. Así, el desarrollo se sitúa y adapta a la cultura de un territorio, siendo éste un elemento invalorable para potenciar el desarrollo económico y social ${ }^{63}$. El desarrollo integral es situado en un lugar, un momento, y en un conjunto de expectativas y temores, sueños y necesidades ${ }^{64}$.

La cultura es una noción tremendamente amplia y ambigua. Sin embargo, desde el enfoque de desarrollo local ésta asume un papel preponderante en la medida que afectará cómo los habitantes interpretan y actúan sobre el desastre, y cómo éstos se sobreponen a dicho suceso. La cultura influirá en las expresiones que asumen las demás dimensiones del desarrollo, puesto que puede explicar los niveles de capital social, al mismo tiempo que puede permear las formas que asumen las IEL. Por otra parte, la cultura mediará las formas que asume el liderazgo político y el cómo funcionan las organizaciones encargadas de promover el desarrollo. También, la cultura influye en la forma que los habitantes se relacionan con el medioambiente.

De esta manera, en un contexto de desastre la cultura encuentra su máximo nivel de expresión

62 Del Socorro, 2000.

63 Radl, 2000.

64 Ibíd.

128 revista invi № 77 / Mayo 2013 / Volumen № 28: 111-136 de los movimientos en las localidades afectadas, siendo fundamental que las políticas de respuesta a los desastres reconozcan el valor de la cultura local y la incorporen en el desarrollo en todos sus ámbitos. Al ser una situación de crisis, los municipios podrán generar acciones de fomento de los valores, historia y tradiciones locales, favoreciendo el encuentro colectivo, el desarrollo del orgullo, el arraigo al territorio y la resiliencia de las comunidades perturbadas. Se priorizará la reconstrucción del patrimonio local, de las iglesias dañadas y de todos los lugares que son un orgullo para la localidad. En la expresión más concreta de la cultura, se podrán generar acciones de fomento al turismo, potenciando la infraestructura hotelera, reconstruyendo el patrimonio arquitectónico y promoviendo la realización de festividades o ferias típicas de las zonas afectadas. Así, la relación cultura y economía se vuelve un aliado para promover el desarrollo de estas localidades.

\section{POLÍTICA}

En una localidad afectada por un desastre, la política y sus principales actores deben ser capaces de dotar de gobernabilidad al territorio ${ }^{65}$, algo fundamental para manejar y actuar frente a los momentos de crisis. La noción de gobernabilidad nos

65 Gallicchio y Camejo, 2005. 
conduce a distinguir a aquellos cargos y organizaciones encargadas de formular, gestionar y dirigir un proyecto colectivo de desarrollo del territorio, específico, autónomo y sustentado en la participación de los actores locales ${ }^{66}$. En un territorio local, como es una comuna, el municipio y sus máximas autoridades (alcalde y concejo municipal) son los principales actores políticos.

De este modo, las municipalidades y especialmente sus máximas autoridades a menudo concentran un alto grado de poder dentro de los territorios, representando por excelencia la dimensión política del desarrollo local. No obstante, dentro de su mandato legal tienen la obligación de promover la participación de la comunidad en la identificación de sus problemas y formulación de estrategias de solución.

Un proyecto de desarrollo colectivo es fundamental en una situación de desastre ya que permitirá identificar el horizonte hacia el cual se pretende avanzar. Como los desastres generan cambio social, es probable que el proyecto de desarrollo preexistente deba ser reformulado en consideración de las nuevas condiciones. La participación de los actores locales como las organizaciones comunitarias, otras organizaciones públicas y de la sociedad civil y los representantes del sector privado resultan claves para la legitimidad y validación de este proyecto. La dimensión política nos conduce a interrogarnos acerca de la distribución y luchas de poder presentes al interior de un territorio, que también se expresan con fuerza en una situación de crisis como los desastres. El factor político a menudo obstaculiza la implementación efectiva de las políticas post-desastre.

Desde el enfoque del desarrollo local, será muy importante verificar la existencia y revalidación del proyecto de desarrollo, impulsando la participación de actores de diversos sectores y organizaciones. Las condiciones de infraestructura del municipio después del desastre serán objeto de preocupación en la medida que se requieren para que la organización funcione y puedan aplicarse los programas pertinentes. Por otra parte, las condiciones emocionales y capacidades de liderazgo de las autoridades locales serán necesarias potenciar para poder guiar a la comunidad hacia el camino del desarrollo local.

\section{AMBIENTAL}

Los desastres y el medioambiente tienen una estrecha relación, en el origen y en sus consecuencias. Por un lado, los movimientos de la naturaleza producen cierto tipo de desastres (terremotos, tsunamis, huracanes, sequías, inundaciones, entre otros), y por otro lado, los desastres dejan huella en

66 Ibíd. 
la naturaleza, por ejemplo, cambiando la geomorfología como es el caso de los grandes terremotos.

Desde el enfoque de desarrollo local, consideraremos al medioambiente como un hábitat en el cual se encuentran los recursos naturales indispensables para la vida humana ${ }^{67}$, como el agua, aire y alimentos. El medioambiente es una condición de vida para la humanidad y la existencia de la sociedad. Pero esto no siempre ha sido un tema sensible, ya que es recién en la segunda mitad del siglo XX que el cuidado del medioambiente se volvió un tema de preocupación, gozando actualmente de alta popularidad ${ }^{68}$.

Desarrollo y medioambiente son conceptos y objetivos complementarios y convergentes ${ }^{69}$. Sobre todo si el concepto de desarrollo se concibe como algo que va mucho más allá que el crecimiento económico; como un proceso de cambio estructural acompañado por un proceso de liberalización individual que tiene como objetivo la satisfacción de las necesidades humanas y el aumento del bienestar no solo de las generaciones presentes sino también de las futuras ${ }^{70}$. En eso consiste la sostenibilidad del desarrollo, en garantizar que las generaciones futuras puedan aprovechar las ventajas y beneficios de los recursos naturales disponibles en el presente.

\footnotetext{
67 Jiménez, 1992

68 López, 1994.

69 Jiménez, 1992

70 Ibíd.
}

130 revista invi № 77 / Mayo 2013 / Volumen N² 28: 111-136
En un contexto de desastre, la relación entre medioambiente y sociedad es objeto de cuestionamientos y críticas, sobre todo en el caso de los desastres inducidos por la acción de los individuos. Los desastres de origen natural nos recuerda la indomabilidad de la naturaleza. Tras un desastre, las políticas debiesen ser capaces de estudiar la disponibilidad actual, calidad y distribución de los elementos vitales para la sobrevivencia humana, como el agua, aire y alimentos. También es relevante evaluar el daño ambiental provocado por el desastre y generar estrategias de apoyo a los afectados, como a los agricultores. Por ejemplo, en el caso de una sequía es fundamental estudiar los costos y formas de dotar de potencialidad productora a los cultivos de la zona.

La especial sensibilidad hacia el medioambiente estimulada por los desastres, configura un buen escenario para la educación de los habitantes y de las unidades productivas acerca de la sostenibilidad del desarrollo de la localidad, incentivando el surgimiento de iniciativas innovadoras en uso sustentable de recursos naturales y energéticos, que permitan sustentar un desarrollo local a largo plazo del cual puedan beneficiarse generaciones futuras. 


\section{Conclusiones}

A lo largo del artículo, hemos propuesto una perspectiva integral para entender los desastres como fenómenos sociales, detallando cómo en cada una de sus fases se develan particulares relaciones entre sociedad y naturaleza. Este modo de comprender los desastres implica contar con una perspectiva más amplia en la forma de abordar los efectos que generan. Postulamos que al concebir los desastres como fenómenos sociales, las estrategias puramente centradas en la reconstrucción material resultan insuficientes para apoyar a las localidades afectadas.

Al descubrir que en los desastres se encuentra el corazón de la idea de cambio social, sugerimos que se configuran como un escenario propicio para impulsar un cambio a través de estrategias de desarrollo. Más específicamente, propusimos que las políticas de respuesta ante desastres deben formularse en base al enfoque de desarrollo local, centrado en las personas, que considera la reconstrucción como un paso necesario e inicial para apoyar a las localidades azotadas por los sucesos negativos.

Finalmente, propusimos cinco dimensiones que forman parte del desarrollo local y que pueden ser trabajadas por las políticas de respuesta ante desastres. Estas son la dimensión social, política, ambiental, económica y cultural. La potencialidad de

ARTíCULO: Potenciando el desarrollo local de comunidades afectadas por desastres / Alicia Cristina Razeto Pavez un enfoque como éste pasa por abarcar de manera integral las necesidades de una población afectada por un desastre, formulándose una estrategia de desarrollo local en base a sus características y potencialidades.

Una de las principales interrogantes que dejamos aquí planteadas son las vinculadas a la necesidad de transitar desde una reflexión conceptual a una empírica y aplicada. Es imperioso formular estrategias que propongan cómo medir estas dimensiones del desarrollo local en localidades afectadas por desastre, de modo de contribuir a generar propuestas concretas para las políticas de respuesta ante desastre.

\section{Bibliografía}

AGUIRRE, Benigno. Los desastres en Latinoamérica: vulnerabilidad y resistencia. Revista Mexicana de Sociología. 66(3): 485-510, 2004. ISSN 0188-2503.

ALBURQUERQUE, Francisco. Reflexiones sobre iniciativas de desarrollo local en Brasil, Argentina y Chile. [En línea]. Comunidad Virtual de Gobernabilidad y Liderazgo. 2006. [Fecha de consulta: 18 junio 2011]. Disponible en: http://www.gobernabilidad.cl/modules.php?name=News\&file=print \&sid=1134.

ARITO, Sandra y JACQUETT, María Mónica. El trabajo social en situaciones de emergencia o desastre. Buenos Aires, Argentina, Espacio. 2005. 84 p. ISBN 9508022043.

revista invi № 77 / Mayo 2013 / Volumen N ํ2 28: 111-136 131 
AROCENA, José. El desarrollo local: un desafío contemporáneo. 2a ed. Montevideo, Uruguay, Taurus - Universidad Católica. 2002. 175 p. ISBN 980-317-077-51.

AUDEFROY, Joel. Desastres y cultura: una aproximación teórica. [En línea]. Revista INVI. 22(60): 133165, agosto 2007. ISSN 0718-8358. Disponible en: http://revistainvi.uchile.cl/index.php/INVI/ article/view/283/928.

BANCO MUNDIAL. Informe sobre el desarrollo mundial. Panorama general. Desarrollo y cambio climático. [En línea]. Banco Mundial. 2010. [Fecha de consulta: 20 marzo 2012]. Disponible en: http:// siteresources.worldbank.org/INTWDR2010/ Resources/5287678-1226014527953/OverviewSpanish.pdf.

BITAR, Sergio. Doce lecciones del terremoto chileno. [En línea]. Estado, Gobierno, Gestión Pública: Revista Chilena de Administración Pública. (15-16): 7-18, 2010. ISSN 0717-8980. Disponible en: http:// www.revistaeggp.uchile.cl/index.php/REGP/ article/viewArticle/11205.

CAPUTO, María Graciela y HERZER, Hilda. Reflexiones sobre el manejo de las inundaciones y su incorporación a las políticas de desarrollo regional. Desarrollo Económico. 27(106): 245-260. 1987. ISSN 0046-001X

CARDONA, A., Omar. Estimación holística del riesgo sísmico utilizando sistemas dinámicos complejos. [En línea]. Red de Estudios Sociales en Prevención de Desastres en América Latina - LA RED. Diciembre

132 revista invi № 77 / Mayo 2013 / Volumen № 28: 111-136
2001. [Fecha de consulta: 10 septiembre 2012]. Disponible en: http://www.desenredando.org/ public/varios/2001/ehrisusd/.

CEPAL y BID. Un tema del desarrollo: la reducción de la vulnerabilidad frente a los desastres. [En línea]. CEPAL. 2000. Disponible en: http://www.eclac. org/publicaciones/xml/4/10134/L428.pdf.

CEPAL. Desastres y desarrollo: el impacto en 2010, cifras preliminares. [En línea]. Unidad de Desastres CEPAL. 2010. [Fecha de consulta: 18 enero 2012]. Disponible en: http://www.eclac.cl/desastres/noticias/noticias/2/42102/Desastres2010_WEB.pdf.

CEPAL. Recomendaciones para una estrategia de reconstrucción y recuperación del terremoto de Chile del 27 de febrero de 2010: borrador para discusión, mayo de 2010. [En línea] Unidad de Evaluación de Desastres CEPAL. [Fecha de consulta: 8 septiembre 2012). Disponible en: http:// www.eclac.cl/publicaciones/xml/4/41564/RECOMENDACIONES_PARA_UNA_ESTRATEGIA_ DE_RECONSTRUCCI\%C3\%93N_Y_RECUPERACION_-_CHILE_MASTERrev6.pdf.

COPPOLA, Damon. Introduction to international disaster management. 2nd. ed. Boston, Butterworth Heinemann. 2011. 696 p. ISBN 978-0-12-382174-4.

DYNES, Russell. Cross-cultural international research: sociology and disaster. [En línea]. International Journal of Mass Emergencies and Disaster. 6(2): 101-129, 1988. ISSN 0280-7270. Disponible en: http://ijmed.org/articles/131/.

ARTíCULO: Potenciando el desarrollo local de comunidades afectadas por desastres /

Alicia Cristina Razeto Pavez 
FURIÓ, Elies. El desarrollo económico endógeno y local: reflexiones sobre su enfoque interpretativo. [En línea]. Estudios Regionales. (40): 97-112, 1994. ISSN 0213-7585. Disponible en: http://www.revistaestudiosregionales.com/pdfs/pdf456.pdf.

GALLICCHIO, Enrique y CAMEJO, Alejandra. Desarrollo local y descentralización en América Latina: nuevas alternativas de desarrollo. Montevideo, Uruguay, Centro Latinoamericano de Economía Humana (CLAEH), Diputación de Barcelona. 2005. 183 p. ISBN: 9974-581-28-1.

GARCíA A., Virginia, coord. Historia y desastres en América Latina, volumen III. [En línea]. México, Centro de Investigaciones y Estudios Superiores en Antropología Social, Red de Estudios Sociales en Prevención de Desastres en América Latina (La Red). 2008. [Fecha de consulta: 15 junio 2011]. Disponible en: http://www.desenredando.org/public/libros/2008/hyd/Historia_y_Desastres_VolumenIII.pdf.

HERZER, Hilda et al. Convivir con el riesgo o la gestión del riesgo. [En línea]. CESAM. 2002. [Fecha de consulta: 15 enero 2012]. Disponible en: http:// www.cesam.org.ar/PDF/Convivir\%20con\%20 el\%20riesgo\%20o\%20la\%20gesti\%C3\%B3n\%20 del\%20riesgo\%20(2002).pdf.

IPCC. Cambio climático 2007: Informe de síntesis. Contribución de los Grupos de trabajo I, II y III al Cuarto Informe de evaluación del Grupo Intergubernamental de Expertos sobre el Cambio Climático. [En línea]. Ginebra, Suiza, IPCC. 2008. 104 p. ISBN 92-9169-322-7. Disponible en: http:// www.ipcc.ch/pdf/assessment-report/ar4/syr/ ar4_syr_sp.pdf.

JIMÉNEZ, Luis. Medioambiente y desarrollo alternativo. 2a ed. Madrid, Iepala. 1992. 445 p. ISBN 84-85436-90-3.

KREPS, Gary. Disaster as a systemic event and social catalyst. En: QUARANTELLI, Enrico. What is a disaster? perspectives on the question. New York, Routledge. 1998. p. 25-50. ISBN 0-203-98483-8.

LAVELL, Allan. Ciencias sociales y desastres naturales en América Latina: un encuentro inconcluso. [En línea]. Eure. 19(58): 73-84, 1993. ISSN 02507161. Disponible en: http://www.eure.cl/numero/ ciencias-sociales-y-desastres-naturales-en-america-latina-un-encuentro-inconcluso/.

LAVELL, Allan. Degradación ambiental, riesgo y desastre urbano: problemas y conceptos. EN: FERNÁNDEZ, María Augusta. Ciudades en riesgo. Degradación ambiental, riesgos urbanos y desastre. Lima, La Red/USAID. 1996. p. 12-42. ISBN 9972-47-001-6.

LAVELL, Allan. Desastres urbanos: una visión global. [En línea]. Red de Estudios Sociales en Prevención de Desastres en América Latina, LA RED. 2000. [Fecha de consulta: 12 enero 2012]. Disponible en: http://www.desenredando.org/public/articulos/2000/duuvg/DUUVG_mar-1-2002.pdf.

LAVELL, Allan. Desastres y desarrollo: hacia un entendimiento de las formas de construcción social de un desastre: el caso del huracán Mitch en Centroamérica. [En línea]. Red de Estudios Sociales

revista invi ํำ 77 / Mayo 2013 / Volumen N² 28: 111-136 133 
en Prevención de Desastres en América Latina, LA RED. 2000. [Fecha de consulta: 25 noviembre 2011]. Disponible en: http://www.desenredando. org/public/articulos/2000/dyd/DyD2000_mar-12002.pdf.

LERNER, Richard. Concepts and theories of human development. 3rd ed, New Jersey, Lawrence Erlbaum Associates, Routledge. 2002. 613 p. ISBN 0-8058-2798-6

LÓPEZ Bonillo, Diego. El medioambiente. España, Cátedra. 1994. 385 p. ISBN 8437612446.

LÓPEZ Tagle, Elizabeth y SANTANA N., Paula. El terremoto de 2010 en Chile: respuesta del sistema de salud y de la cooperación internacional. [En línea]. Revista Panamericana de Salud Pública. 30(2): 160-166. 2011. ISSN 1020-4989. Disponible en: http://www.scielosp.org/scielo.php?script=sci_ arttext $\&$ pid $=S 1020-49892011000800008 \& \operatorname{lng}=$ en\&nrm=iso\&tlng=es

MANSILLA, Elizabeth. Desastres y desarrollo en México. [En línea]. Desastres y Sociedad. 1(1): 2-12. 1993. Disponible en: http://www.desenredando.org/public/revistas/dys/rdys01/dys1-DDMoct-24-2001.pdf.

MARTín Ríos, Diego. Urbanización de áreas inundables, mediación técnica y riesgo de desastre: una mirada crítica sobre sus relaciones. [En línea]. Revista de Geografía Norte Grande. (47): 27-43. 2010 ISSN 0718-3402. Disponible en: http://dx.doi org/10.4067/S0718-34022010000300002.
MCCAUGHEY, Brian; HOFFMAN, Kenneth y LLEWELLYN, Criag. The human experience of earthquakes. En: INDIVIDUAL and community responses to trauma on disaster. New York, Cambridge University Press. 1995. p. 136-153. ISBN10: 0521556430

MITCHELL, James K., ed. Crucibles of hazards: megacities and disasters in transition. Tokyo, United Nations University Press. 1999. 450 p. ISBN-10: 9280809873.

MOTA, Laura y DÍAZ, Pablo. Municipios, desarrollo local y descentralización en el siglo XXI. [En línea]. Ra Ximhai Revista Sociedad, Cultura y Desarrollo Sustentable. 4(3): 581-605, 2008. ISSN 16650441. Disponible en: http://www.ejournal.unam. mx/rxm/vol04-03/RXM004000304.pdf.

OBSERVATORIO de la Reconstrucción. Noticias. [En línea]. (Observatorio de la reconstrucción). 2011. [Fecha de consulta: 20 enero 2012]. Disponible en: http://reconstruccion.uchilefau.cl/?cat=3.

OEA. Desastres, planificación y desarrollo: manejo de amenazas naturales para reducir los daños. [En línea]. Organization of American States. 1991. [Fecha de consulta: 9 marzo 2012]. Disponible en: http:// www.oas.org/dsd/publications/Unit/oea57s/ oea57s.pdf.

OLIVERA, Andrés y GONZALEZ Gonzalo. Enfoque multidimensional de la reconstrucción post-desastre de la vivienda social y el hábitat en países en vías de desarrollo: estudios de casos en Cuba. [En línea]. Revista de la Construcción. 9(2): 53-62, 2010.

ARTíCULO: Potenciando el desarrollo local de comunidades afectadas por desastres /

Alicia Cristina Razeto Pavez 
ISSN 0718-915X. Disponible en: http://dx.doi. org/10.4067/S0718-915X2010000200006.

OLIVER-SMITH, Anthony. Anthropological research on hazards and disasters. [En línea]. Annual Review of Anthropology. (25): 303-328. 1996 ISSN 0084-6570. Disponible en: http://dx.doi. org/10.1146/annurev.anthro.25.1.303.

OLIVER-SMITH, Anthony. Perspectivas antropológicas en la investigación de desastres. [En línea]. Desastres y Sociedad. 3(5): 53-74. 1995. Disponible en: http://www.desenredando.org/public/revistas/ dys/rdys05/dys5-1.0-paeid.pdf.

ORGANIZACIÓN PANAMERICANA DE LA SALUD. Hacia un mundo más seguro frente a los desastres naturales. La trayectoria de América Latina y el Caribe. [En línea]. Organización Panamericana de la Salud. 1994. [Fecha de consulta: 13 junio 2012]. Disponible en: http://www.paho.org/spanish/Ped/ws-toc_capitulol.pdf.

PERRY, Ronald. What is a disaster? En: HANDBOOK of disaster research. New York, Springer. 2007. p. 1-15. ISBN 978-0-387-73952-6.

PLIEG0, Fernando. Estrategias de desarrollo social en situaciones de desastre. Revista Mexicana de Sociología. 54(4): 11-24, 1992. ISSN 0188-2503.

QUARANTELLI, Enrico. Urban vulnerability to disasters in developing countries: managing risks. En: BUILDING safer cities: the future of disaster risk. Washington, DC, The World Bank. 2003. p. 211232. ISBN 0-8213-5497-3
RODRÍGUEZ, Daniel. Desastre y vulnerabilidad. Entre las ciencias naturales y las ciencias sociales. En: LOS DESASTRES en México: una perspectiva multidisciplinaria. México, UIA/UNAM. 1998. p. 19-38. ISBN 968-859-329-X.

ROMERO, Hugo; FUENTES, Claudio y SMITH, Pamela. La geografía de los riesgos "naturales" y el terremoto de Chile del 27 de febrero de 2010. En: GEOGRAFÍA y ambiente en América Latina. México DF, UNAM, CIGA, Semarnat, INE. 2011. p. 251-282. ISBN 6070224965.

SANZANA Calvet, Martín. Desastre natural y acción colectiva de los sectores populares en Chile: los saqueos en Concepción tras el 27/F. [En línea]. Revista Observatorio Social de América Latina OSAL. XI (28): 145-158, noviembre 2010. ISSN 1515-3282. Disponible en: http://biblioteca.clacso.edu.ar/ar/libros/osal/osal28/09Sanzana.pdf.

SMITH, Gavin y WENGER, Dennis. Sustainable disaster recovery: operationalizing an existing agenda. En: HANDBOOK of disaster research. New York, Springer. 2007. p. 234-257. ISBN 978-0-387-32331-2.

STEPHENSON, R.S. Logística. [En línea]. Disaster Info. 1991. [Fecha de consulta: 6 agosto 2012). Disponible en: http://www.disaster-info.net/lideres/ spanish/mexico/biblio/spa/doc13949.pdf.

TUCKER, Brian E.; ERDIK, Mustafa y HWANG, Christina N. Issues in urban earthquake risk. USA, Kluwer. 1994. 329 p. ISBN-10 0792329147. 
VACHON, Bernard. La práctica del desarrollo local. En: VACHON, Bernard; COALLIER, Francine y RODRÍGUEZ, Fernin. El desarrollo local. Teoría y práctica. Reintroducir lo humano en la lógica del desarrollo. España, Ediciones Trea. 2001. ISBN 9788495178978.

VAN AALST, Maarten. The impacts of climate change on the risk of natural disasters. [En línea]. Disaster, 30(1): 5-18, 2006. ISSN 1467-7717. Disponible en: http://dx.doi. org/10.1111/j.1467-9523.2006.00303.x.

VARGAS, Jorge. Políticas públicas para la reducción de la vulnerabilidad frente a los desastres naturales y socio-naturales. [En línea]. Santiago, CEPAL. 2002. ISBN: 92-1-322013-8. Serie Medioambiente y Desarrollo No50. Disponible en: http://www. eclac.org/cgi-bin/getProd.asp?xml=/publicaciones/xml/1/10561/P10561.xml\&xsl=/dmaah/tpl/ p9f.xsl\&base=/dmaah/tpl/top-bottom.xsl.

WISNER, Blaikie et al. At risk natural hazards people's vulnerability and disasters. New York, Routledge. 2004. 471 p. ISBN 0-415-25216-4. 